\title{
Model Describing Material-Dependent Deformation Behavior in High Velocity Metal Forming Processes
}

\author{
Håkan Hallberg $\dagger^{*}$, Kristina Ryttberg $\ddagger$ and Matti Ristinmaa† \\ †Division of Solid Mechanics \\ Lund University, Box 118, S-221 00 Lund, Sweden \\ $\ddagger$ Department of Materials and Manufacturing Technology \\ Chalmers University of Technology, S-412 96 Göteborg, Sweden \\ *hakan.hallberg@solid.Ith.se
}

\begin{abstract}
A constitutive model for rate dependent and thermomechanically coupled plasticity at finite strains is presented. The plasticity model is based on $J_{2}$ plasticity and rate dependent behavior is included by use of a Perzyna-type formulation. Adiabatic heating effects are handled in a consistent way and not, as is a common assumption, through a constant conversion of the internal work rate into rate of heating. The conversion factor is instead derived from thermodynamic considerations. The stored energy is assumed to be a function of a single internal variable which differs from the effective plastic strain. This allows a thermodynamically consistent formulation to be obtained which, as shown, can be calibrated by use of simple procedures. Choosing 100Cr6 steel in two differently heat treated conditions as prototype material, experimental tests are performed enabling the model to be calibrated. Significant differences in deformation behavior are noted as the differently heat treated specimens are compared. In addition, the local stress-updating procedure is reduced to a single scalar equation, permitting a very efficient numerical implementation of the model. The constitutive formulation proposed was employed in an explicit finite element solver, illustrative simulations of a high velocity metal forming process being performed to demonstrate the capabilities of the model and certain characteristic traits of the
\end{abstract}

DOI: $10.1061 /$ ASCE0733-93992009135:4345 
ASCE Journal of Engineering Mechanics 2009, 135(4), 345-357

materials that were studied.

Keywords: Constitutive models, viscoplasticity, continuum mechanics, experimental results

\section{Introduction}

During the plastic deformation of a metallic material, a major part of the internal plastic work is converted to, or dissipated as, heat. The remaining, smaller, portion of this energy is stored in the material through the creation and rearrangement of crystal imperfections. These imperfections can consist of dislocations, point defects, stacking faults and twins, cf. Bever et al. (1973), Oliferuk et al. (1985) and Rosakis et al. (2000). If the plastic deformation is achieved fast enough that no heat exchange with the surroundings is possible - which in high speed metal forming operations is the common case - the heating is of adiabatic nature. The conversion of internal plastic work into heat has, in line with the early work of Taylor and Quinney (1934), traditionally been handled in numerical simulations by converting a fixed amount - typically 80-95\% - of the rate of plastic work into internal heating. It has been concluded, however, that this heat exchange ratio and correspondingly the evolving stored energy of cold work - is not a fixed number but instead is a variable that depends on both the accumulated strain and the strain rate. Numerous authors have conducted both experimental and theoretical studies regarding this phenomenon, e.g. Chrysochoos et al. (1989); Mason et al. (1994); Kapoor and NematNasser (1998); Hodowany et al. (2000); Rosakis et al. (2000); Håkansson et al. (2005); Ristinmaa et al. (2007). In particular, the earlier comprehensive review work by Bever et al. (1973) in which experimental methods and results regarding the stored energy of cold work are discussed should be mentioned.

When the deformation of metals and steel gradually increases, the heat generated by the dissipation of the irreversible internal work performed leads to a pronounced thermal softening of the material. This effect can gradually counteract and reverse the deformation hardening that also occurs. In the presence of geometrical or material irregularities, this softening can also promote the initiation and growth of shear bands. Within these very narrow regions of highly localized plastic deformation, the temperature increases further, supporting additional thermal softening and localization. This localization tendency is especially pronounced when adiabatic conditions prevail. High speed deformation has been studied by many authors, such as Trötzschel et al. (1997) and in connection with heat generation in adiabatic shear bands by Clifton et al. (1984); Hartley et al. (1987); Marchand and Duffy (1988); Batra and Liu (1990); Zhu and Batra (1992); Tugcu (1995) and Batra and Stevens (1998). 
Steel exposed to loading, sufficient to induce plasticity in the material, exhibits responses that depend upon the loading rate. In numerical simulations, viscoplastic formulations are commonly used to describe this behavior. In the present paper, the widely used Perzyna approach is adopted for the rate dependent part of the model, cf. Perzyna (1966) and Olszak and Perzyna (1969). The Perzyna format has found wide use, model formulations involving it having been taken up in several studies of viscoplasticity, for example Phillips and $\mathrm{Wu}$ (1973); Ristinmaa and Ottosen (2000); Heeres et al. (2002) and Runesson et al. (1999). The present model is set in a thermodynamically consistent continuum mechanical formulation. A multiplicative split of the deformation gradient into both a reversible, i.e. elastic and thermal, and an irreversible, i.e. viscoplastic, part is used to facilitate a finite strain formulation. The hardening behavior of the model is controlled by the effective plastic strain whereas the stored energy is assumed to be governed by a single internal variable, one that differs from the effective plastic strain. This allows a formulation to be employed in which the stored energy of cold work, that enters the Helmholtz energy function, can be determined in a single uniaxial experiment. This in turn is found to completely determine additional components of the constitutive model, such as the hardening potential, the non-associated yield condition and the non-constant heat exchange ratio.

The present paper begins with definitions of the finite strain kinematics and the thermodynamic framework used in the present model. The constitutive model is then obtained through a specific choice of the form of the Helmholtz energy function. The quantities to be determined on the basis of experiments are defined. The specific experimental procedures and the calibration of the model are described in the section that follows. A $100 \mathrm{Cr} 6$ steel was chosen as a prototype material, uniaxial tension tests being performed on it at different temperatures and strain rates. Heating through internal dissipation is measured approximately by recording the temperature increase at the surface of the tensile specimens. The experimental data obtained allows the proposed constitutive model to be calibrated. The heat exchange ratio involved in the model is then described. A presentation of the results and a concluding discussion completes the main part of the paper. The numerical implementation scheme concerned with integration of the constitutive relations is included in appendix A, in which the local stress-updating procedure is reduced to involve only a single scalar equation, expressed in terms of the effective viscoplastic strain. This allows a very efficient numerical implementation of the model in a commercial, explicit, finite element package that is used to perform illustrative simulations of a high velocity forming process.

\section{Preliminaries}

The rate of deformation tensor $\boldsymbol{d}$ can be expressed as $\boldsymbol{d}=\operatorname{sym}(\boldsymbol{l})$. In this expression, the notation $\operatorname{sym}(\cdot)$ is introduced to indicate the symmetric part of a tensorial quantity and $\boldsymbol{l}$ denotes the spatial velocity gradient given by $\boldsymbol{l}=\dot{\boldsymbol{F}} \boldsymbol{F}^{-1}$, where $\boldsymbol{F}$ denotes the deformation 
gradient. The superposed dot here denotes the material time derivative and $(\cdot)^{-1}$ the inverse. To allow for the modeling of an inelastic response, a multiplicative split of the deformation gradient into a reversible part and an irreversible part is adopted, cf. Kröner (1960) and Lee and Liu (1967). The resulting decomposition is then given as

$$
\boldsymbol{F}=\boldsymbol{F}^{r} \boldsymbol{F}^{v p}, \quad \boldsymbol{F}^{r}=\boldsymbol{F}^{\theta} \boldsymbol{F}^{e}
$$

where the superscripts $r$ and $v p$ indicate the reversible, i.e. elastic and thermal, and the viscoplastic, i.e. irreversible, components, respectively. The superscripts $e$ and $\theta$, in turn denote the elastic and the thermal components, respectively. $\boldsymbol{F}^{r}$ is thus the fully recoverable thermoelastic deformation which is superimposed on the inelastic - i.e. viscoplastic component $\boldsymbol{F}^{v p}$. In accordance with the spatial velocity gradient, the following notations are introduced for the reversible and the irreversible part of the velocity gradient, respectively: $\boldsymbol{l}^{r}=\dot{\boldsymbol{F}}^{r} \boldsymbol{F}^{r-1}$ and $\boldsymbol{L}^{v p}=\dot{\boldsymbol{F}}^{v p} \boldsymbol{F}^{v p-1}$. Using the previous multiplicative split of the deformation gradient allows one to write

$$
\boldsymbol{l}=\dot{\boldsymbol{F}}^{r} \boldsymbol{F}^{r-1}+\boldsymbol{F}^{r} \boldsymbol{L}^{v p} \boldsymbol{F}^{r-1} \equiv \boldsymbol{l}^{r}+\boldsymbol{l}^{v p}
$$

where the viscoplastic spatial velocity gradient is defined as $\boldsymbol{l}^{v p}=\boldsymbol{F}^{r} \boldsymbol{L}^{v p} \boldsymbol{F}^{r-1}$. Note in addition that the reversible part of the Finger tensor can be expressed as

$$
\boldsymbol{b}^{r}=\boldsymbol{F}^{r} \boldsymbol{F}^{r T}
$$

Differentiation with respect to time and using (2) gives

$$
\dot{b}^{r}=\boldsymbol{l} \boldsymbol{b}^{r}+\boldsymbol{b}^{r} \boldsymbol{l}^{T}-\left(\boldsymbol{l}^{v p} \boldsymbol{b}^{r}+\boldsymbol{b}^{r} \boldsymbol{l}^{v p T}\right)
$$

Let $\psi(\boldsymbol{A}, \theta)$ denote the Helmholtz energy with $\boldsymbol{A} \in\left(\boldsymbol{b}^{r}, \kappa\right)$ being the set of internal variables under consideration here. The effects of plasticity in the material is described through the internal variable $\kappa$. Assuming adiabatic conditions, the heat equation can then be written as

$$
\rho_{0} c \dot{\theta}=\boldsymbol{\tau}: \boldsymbol{d}-\rho_{0} \frac{\partial \psi}{\partial \boldsymbol{A}}: \dot{\boldsymbol{A}}+\rho_{0} \theta \frac{\partial^{2} \psi}{\partial \theta \partial \boldsymbol{A}}: \dot{\boldsymbol{A}}
$$

where $\rho_{0}$ is the mass density in the reference configuration and $c=-\theta \partial^{2} \psi / \partial \theta^{2}$ is the specific heat capacity. The last term in (5) contains components related to the thermoelastic behavior of the material. For continuum mechanical purposes, and under adiabatic conditions, the second law of thermodynamics can be written as a dissipation inequality on the format

$$
\mathcal{D}=\boldsymbol{\tau}: \boldsymbol{d}-\rho_{0} \frac{\partial \psi}{\partial \boldsymbol{A}}: \dot{\boldsymbol{A}} \geq 0
$$


where $\mathcal{D}$ denotes the dissipation. By taking advantage of $\boldsymbol{A} \in\left(\boldsymbol{b}^{r}, \kappa\right)$ and using (4), a reformulation can be made, allowing the mechanical dissipation to be defined as

$$
\mathcal{D}_{\text {mech }}=\boldsymbol{\tau}: \boldsymbol{d}^{v p}-R \dot{\kappa} \geq 0
$$

where $\boldsymbol{d}^{v p}=\operatorname{sym}\left(\boldsymbol{l}^{v p}\right)$ denote the viscoplastic strain rate tensor. In (7), the fact that the Kirchhoff stress tensor can be formulated as

$$
\boldsymbol{\tau}=2 \rho_{0} \boldsymbol{b}^{r} \frac{\partial \psi}{\partial \boldsymbol{b}^{r}}
$$

and that $\psi$ is an isotropic function of $\boldsymbol{b}^{r}$, i.e. that $\partial \psi / \partial \boldsymbol{b}^{r}$ and $\boldsymbol{b}^{r}$ commute, is utilized. In addition, the thermodynamic force $R$ conjugated to $\kappa$ is defined as

$$
R=\rho_{0} \frac{\partial \psi}{\partial \kappa}
$$

This term is related to the stored energy-component of the Helmholtz energy function, a matter discussed in the next section.

\section{The constitutive model}

For steel, which is considered here, the plastic deformation that occurs is assumed to be completely isochoric, i.e. $J^{p}=\operatorname{det}\left(\boldsymbol{F}^{p}\right)=1$, and the thermal deformation to be purely volumetric, i.e. $\boldsymbol{F}^{\theta}=\left(J^{\theta}\right)^{\frac{1}{3}} \mathbf{1}$, where $\mathbf{1}$ is the second-order identity tensor. Note that due to the assumption of plastic incompressibility it holds that $J=\operatorname{det}(\boldsymbol{F})=J^{r}$. The isochoric part of the reversible Finger tensor can be obtained then as

$$
\boldsymbol{b}_{i}^{e}=\boldsymbol{b}_{i}^{r}=J^{-\frac{2}{3}} \boldsymbol{b}^{r}
$$

where the subscript $i$ is introduced to denote isochoric quantities. A specific form of the Helmholtz energy function needs to be used in defining the constitutive model. Assuming a linear temperature dependence of the specific heat $c$ such that $c=c_{1}+c_{2}\left(\theta-\theta_{0}\right)$, where $c_{i}$ are parameters and $\theta_{0}$ the reference temperature, and assuming that the instantaneous elastic response does not depend on the internal variable $\kappa$, cf. Lubliner (1972) and Ottosen and Ristinmaa (2005), an additative decomposition of the Helmholtz energy can be made. The components consist of an elastic part $\psi^{e}\left(\boldsymbol{b}^{r}, \theta\right)$ which can be linear in $\theta$, a thermal part $\psi^{\theta}(\theta)$ and a viscoplastic part $\psi^{v p}(\kappa)$ which is related to the stored energy. The Helmholtz energy function now appears as

$$
\psi\left(\boldsymbol{b}_{i}^{e}, J^{r}, \kappa, \theta\right)=\psi^{e}\left(\boldsymbol{b}_{i}^{e}, J^{r}, \theta\right)+\psi^{v p}(\kappa)+\psi^{\theta}(\theta)
$$

In the present model the components of the Helmholtz energy function are taken as

$$
\begin{aligned}
\psi^{e} & =K\left[\frac{1}{2}\left(\ln J^{r}\right)^{2}-3 \alpha\left(\theta-\theta_{0}\right) \ln J^{r}\right] \frac{1}{\rho_{0}}+2 G J_{2}^{r} \frac{1}{\rho_{0}} \\
\psi^{\theta} & =\left[\left(c_{1}-c_{2} \theta_{0}\right) \theta(1-\ln \theta)-\frac{1}{2} c_{2} \theta^{2}\right]
\end{aligned}
$$


where

$$
J_{2}^{r}=\frac{1}{2} \ln \boldsymbol{V}_{i}^{r}: \ln \boldsymbol{V}_{i}^{r}=\frac{1}{2}\left(\ln \boldsymbol{V}^{r}\right)^{d e v}:\left(\ln \boldsymbol{V}^{r}\right)^{d e v}
$$

In (12), $\alpha$ denotes the coefficient of thermal expansion, $K$ the bulk modulus and $G$ the shear modulus. In (13) in addition, $\boldsymbol{V}^{r}=\sqrt{\boldsymbol{b}^{r}}$. The particular form of $\psi^{v p}$ to be used will be discussed later on in connection with calibration of the stored energy of cold work. The above choice of energy function allows the Kirchhoff stress tensor to be obtained from (8) as

$$
\boldsymbol{\tau}=K\left[\ln \left(J^{r}\right)-3 \alpha\left(\theta-\theta_{0}\right)\right] \mathbf{1}+2 G \ln \left(\boldsymbol{V}^{r}\right)^{\operatorname{dev}}
$$

Assuming a stress-free response for $\boldsymbol{F}^{r}=\boldsymbol{F}^{\theta}$, it follows that volumetric expansion due to a change in temperature can be identified as $J^{\theta}=\exp \left[3 \alpha\left(\theta-\theta_{0}\right)\right]$.

For defining the viscoplastic part of the model, advantage is taken of the approach put forward by Ristinmaa et al. (2007) in which it was shown that adopting a non-associated formulation for the internal variable allows other variables than the internal variable to be used in the yield function. Accordingly, in the present model $\kappa$ is employed in both the Helmholtz energy function and in the potential function defining the evolution law whereas in the yield function the effective viscoplastic strain $\varepsilon_{\mathrm{eff}}^{v p}$ is adopted instead. It is first assumed that the von Mises yield function

$$
f=\sigma_{\mathrm{eff}}-\sigma_{\mathrm{y}}\left(\varepsilon_{\mathrm{eff}}^{v p}, \theta\right)
$$

defines the elastic region, i.e. $f \leq 0$, and also that the yield stress is given by

$$
\sigma_{\mathrm{y}}\left(\varepsilon_{\mathrm{eff}}^{v p}, \theta\right)=\hat{\sigma}_{\mathrm{y}}\left(\varepsilon_{\mathrm{eff}}^{v p}\right) T(\theta)
$$

where $\sigma_{\mathrm{y}}(0, \theta)$ can be identified as the initial yield stress. The function $T(\theta)$ that describes the nonlinear dependence of the yield stress on temperature also satisfies that $T\left(\theta_{0}\right)=1$. The hardening of the material is described by the function $\hat{\sigma}_{\mathrm{y}}\left(\varepsilon_{\text {eff }}^{v p}\right)$. Both $T$ and $\hat{\sigma}_{\mathrm{y}}$ are determined on the basis of the response of the material as found by experimental tests. This multiplicative decomposition of the yield stress into components related to hardening and temperature, respectively, is motivated by the activation energy for plastic flow being dependent only on temperature and not on the state of stress or strain in the material, cf. Sherby et al. (1957); Miller (1987). In addition, the effective stress appearing in (15) is defined as

$$
\sigma_{\mathrm{eff}}=\left(\frac{3}{2} \boldsymbol{\tau}^{d e v}: \boldsymbol{\tau}^{d e v}\right)^{\frac{1}{2}}
$$

In order to fulfill the dissipation inequality, it is postulated that the evolution laws are governed by a convex potential function $g(\boldsymbol{\tau}, R, \theta)$ of $\boldsymbol{\tau}$ and $R$ such that $g(\boldsymbol{\tau}, R, \theta)-$ 
$g(\mathbf{0}, 0, \theta) \geq 0$. Whereas $\sigma_{\mathrm{y}}$ describes the mechanical response of the material, $R$ controls the dissipative traits of the model and thus the heat generation due to internal viscoplastic work. Introducing $\lambda$ as a viscoplastic multiplier allows the following evolution laws to be obtained, leading to a non-associated formulation

$$
\begin{aligned}
& \boldsymbol{d}^{v p}=\lambda \frac{\partial g}{\partial \boldsymbol{\tau}} \\
& \dot{\kappa}=-\lambda \frac{\partial g}{\partial R}
\end{aligned}
$$

The particular form of the potential function considered here is given by

$$
g=\sigma_{\text {eff }}-g_{R}(R, \theta)
$$

indicating the evolution law for the plastic strain rate to be associated whereas the evolution law for the internal variable is, generally, non-associated. The specific form of $g_{R}$ will be discussed later in relation to stored energy. Taking advantage of (18) allows the rate of effective viscoplastic strain to be identified as

$$
\dot{\varepsilon}_{\mathrm{eff}}^{v p}=\left(\frac{2}{3} \boldsymbol{d}^{v p}: \boldsymbol{d}^{v p}\right)^{\frac{1}{2}} \equiv \lambda
$$

where

$$
\boldsymbol{d}^{v p}=\lambda \frac{3}{2} \frac{\boldsymbol{\tau}^{\mathrm{dev}}}{\sigma_{\mathrm{eff}}}
$$

A Perzyna formulation is adopted to include viscous effects in the model, the viscoplastic multiplier $\lambda$ being defined as

$$
\lambda=\frac{1}{\eta} h(f)
$$

where $\eta$ is a viscosity parameter and $h$ an overstress function, as originally proposed by Perzyna (1966) and Olszak and Perzyna (1969). In the present model, an overstress function of power-law type is chosen as

$$
h=\left\langle\frac{f}{\sigma_{\mathrm{y}}(0, \theta)}\right\rangle^{n}
$$

where the McCauley-brackets $\langle x\rangle=\frac{1}{2}(x+|x|)$ denote a ramp function for any scalar $x$, and where $\sigma_{\mathrm{y}}$ is defined as in (16). Due to convexity requirements, the exponent $n$ has to fulfill $n \geq 1$, cf. Simo and Hughes (2000). 


\section{Experimental results and model calibration}

To calibrate the model different experimental procedures were performed on $100 \mathrm{Cr} 6$ steel to determine the material behavior at different temperatures and loading rates. The steel was tested in two different heat treatment conditions: as-received spheroidize-annealed material (SA) having a microstructure of ferrite containing spheroidized carbides and also a material that was quenched and tempered (QT) at $610^{\circ} \mathrm{C}$ for 120 min resulting in a martensitic microstructure with a hardness of $340 \mathrm{HV}$. Results for the material response at very high strain rates $\left(1.1-2.0 \times 10^{3} \mathrm{~s}^{-1}\right)$ were taken from Ryttberg (2007), the experiments having been performed using a Split Hopkinson Pressure Bar. These experiments had all been carried out at room temperature. The SA material tested in this way had a hardness of $256 \mathrm{HV}$. Tests at a lower range of loading rates were performed in the present work as standard tensile tests using round bars with initial diameter $d_{0}=7 \mathrm{~mm}$ and an initial gage length $l_{0}=10 d_{0}$, cf. Fig. 1 . Some of the tension tests were conducted at elevated temperatures by encapsulating the specimen in a furnace. The tensile tests were in case of the SA material performed on a material condition having a hardness of $170 \mathrm{HV}$. The experimental results show that, at low strain rates, the rate dependence of the material is relatively small. The hardening behavior of each of the heat treatment conditions can be viewed as basically constant, at least for the range of strain rates indicated in Fig. 1. A Perzyna-type model - i.e. a rate dependent scaling of the yield stress under unchanged hardening conditions - thus appears to provide a close approximation of the viscous behavior of the material.

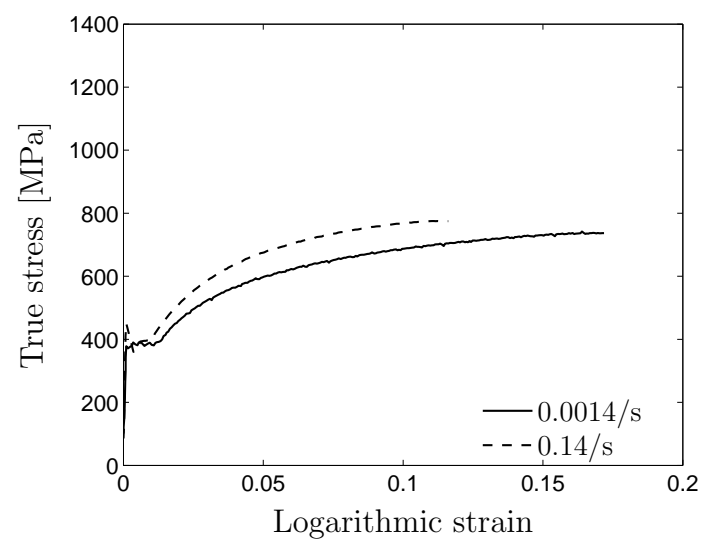

(a) SA

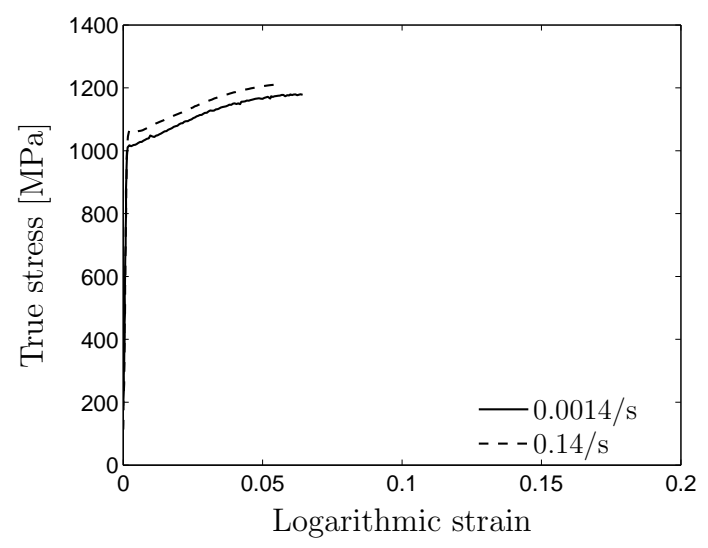

(b) QT

Figure 1: Results of the tensile testing of differently heat treated specimens of $100 \mathrm{Cr} 6$ at two different rates of loading. 


\subsection{Rate dependence}

Employing the Perzyna format, as given by (22), allows the viscous description of the material to be obtained through use of a simple procedure for calibrating the $\eta$ and $n$ material parameters. Recall that in the uniaxial case, according to (20), the rate of viscoplastic strain is equivalent to the viscoplastic multiplier, i.e. $\dot{\varepsilon}_{\text {eff }}^{v p}=\lambda$. The plastic multiplier can also be expressed in terms of the Perzyna overstress function on the basis of relations (22) and (23). This allows the following construction to be made:

$$
\dot{\varepsilon}_{\mathrm{eff}}^{v p}=\frac{1}{\eta}\left\langle\frac{f}{\sigma_{\mathrm{y}}(0, \theta)}\right\rangle^{n} \Rightarrow f=\sigma_{\mathrm{y}}(0, \theta)\left(\eta \dot{\varepsilon}_{\mathrm{eff}}^{v p}\right)^{\frac{1}{n}}
$$

Taking advantage of (15) and (16) also enables one to define a dynamic yield condition as

$$
f^{d}=\sigma_{\mathrm{eff}}-\left[\hat{\sigma}_{\mathrm{y}}\left(\varepsilon_{\mathrm{eff}}^{v p}\right)+\hat{\sigma}_{\mathrm{y}}(0)\left(\eta \dot{\varepsilon}_{\mathrm{eff}}^{v p}\right)^{1 / n}\right] T(\theta)=0
$$

where the last term can be interpreted as a dynamic contribution to the yield stress. For calibration purposes, this allows an initial dynamic yield stress to be identified as

$$
\sigma_{\mathrm{y} 0}^{d}\left(\dot{\varepsilon}_{\mathrm{eff}}^{v p}, \theta\right)=\hat{\sigma}_{\mathrm{y}}(0)\left[1+\left(\eta \dot{\varepsilon}_{\mathrm{eff}}^{v p}\right)^{\frac{1}{n}}\right] T(\theta)
$$

where the superscript $d$ indicates a "dynamic" quantity. Note that under quasi-static conditions, i.e. at very low rates of loading, $f^{d} \approx f$ and $\sigma_{\mathrm{y} 0}^{d}$ approach the quasi-static yield stress $\sigma_{\mathrm{y}}(0, \theta)$. As can be seen in Fig. 1, the initial yield stress at room temperature is $\hat{\sigma}_{\mathrm{y}}(0)=380 \mathrm{MPa}$ for the SA material and $\hat{\sigma}_{\mathrm{y}}(0)=1025 \mathrm{MPa}$ for the QT material. Expressing the dynamic initial yield stress in terms of the rate of viscoplastic strain, allows the rate dependence referred to in (26) - that of $\eta$ and $n$ - to be calibrated for different loading rates against the initial yield stresses that the experimental results provide. The calibration obtained is shown in Fig. 2, where it is assumed that the elastic part of the strain rate is small, i.e. that $\dot{\varepsilon}^{v p} \approx \dot{\varepsilon}$. The general behavior of each curve in Fig. 2 is recognized from e.g. Manjoine (1944). The graphs in Fig. 2 were obtained by choosing $\eta=1 \times 10^{-3} \mathrm{~s}$ and $\eta=5.5 \times 10^{-5} \mathrm{~s}$ for the SA and QT materials, respectively. The exponent $n=2.5$ in the overstress function was chosen for both materials.

\subsection{Temperature dependence}

Encapsulating the tensile specimens in a heating furnace allowed additional tests to be performed on the SA material at elevated temperatures. The temperature dependence of the initial yield stress can be identified from (16) as

$$
\sigma_{\mathrm{y} 0}(\theta)=\hat{\sigma}_{\mathrm{y}}(0) T(\theta)
$$




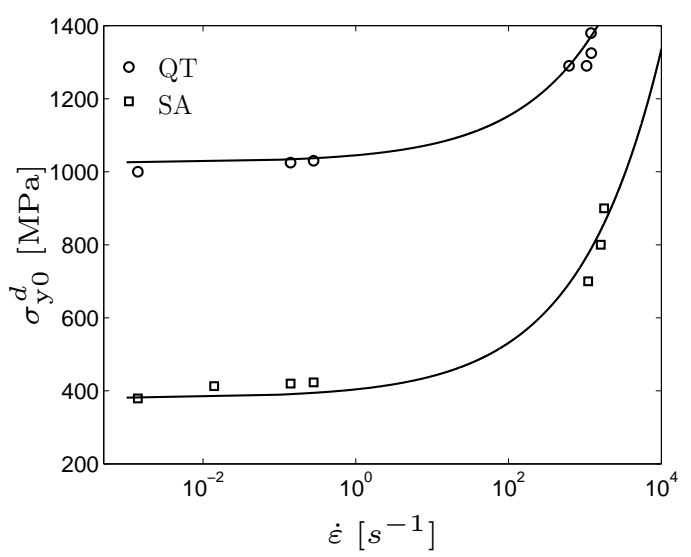

Figure 2: Calibration of the rate dependent initial yield stress of the model (solid lines) against data from experiments (symbols). Note the logarithmic scale on the horizontal axis.

Experimental data displaying the initial yield stress is shown in Fig. 3. To model the drop in initial yield stress as obtained experimentally for the SA material at approximately $700 \mathrm{~K}$, the function $T$ found in (27) was chosen as

$$
T(\theta)=[1-m(\theta)] T_{1}(\theta)+m(\theta) T_{2}(\theta)
$$

where $T_{1}$ and $T_{2}$ are linear functions of the form

$$
T_{1}(\theta)=t_{1} \theta+1 \quad \text { and } \quad T_{2}(\theta)=t_{2} \theta+t_{3}
$$

Expressions $T_{1}$ and $T_{2}$ are combined by use of the function $m$, which has the form

$$
m(\theta)=m_{1}+\frac{1}{\pi}\left[\arctan (\theta-\hat{\theta}) m_{2}\right]
$$

For the SA material suitable choices of the parameters for the linear functions (29) were found to be $t_{\alpha}=\left[0,-1.18 \times 10^{-4} 1 / \mathrm{K},-0.092\right]$ whereas appropriate parameters for $(30)$ were found to be $m_{\alpha}=[0.349,0.005]$ and $\hat{\theta}=690 \mathrm{~K}$. The results in Fig. 3 were obtained using these parameters. This figure also presents results for spheroidized 100Cr6 that were taken from Löwisch and Dalgic (2006). These authors reported $1600 \mathrm{~K}$ to be the approximate absolute melting point of the material.

Unfortunately, experimental data at elevated temperatures for the QT material was not available. Instead, it was assumed that the format given by (28)-(30) can be used to describe the QT-material. The shape of the QT-curve in Fig. 3 was estimated by fitting the simulated force response to the data found from upsetting of cylindrical billets, i.e. to a global response of the material, cf. Fig. 8. Hence it should be noted that the QT-curve in 
Fig. 3 is one possible form that yields a desired localization behavior as seen in experiments, cf. Fig. 9, and is not based on material testing.

It was assumed that since the two materials are essentially of the same composition, their responses at high temperature conditions coincide. In addition, since the initial yield stress at room temperature was known, the fitting procedure revealed the temperature at which the abrupt drop in initial yield stress occurred, i.e. the value of $\hat{\theta}$. This will be discussed later in greater detail. For the QT material, suitable parameter choices were found to be $t_{\alpha}=\left[-6.83 \times 10^{-5} 1 / \mathrm{K},-5.07 \times 10^{-5} 1 / \mathrm{K}, 0.017\right], m_{\alpha}=[0.454,0.026]$ and $\hat{\theta}=770 \mathrm{~K}$. Note that a similar drop in initial yield stress is reported by Shi and Liu (2006) for an equivalent material, heat treated to a hardness of $62 \mathrm{HRC}$. The high-temperature response of the QT material can be affected by the sensitivity of its martensitic structure to elevated temperatures. Since the development of a martensitic phase occurs without any time-consuming diffusion of carbon atoms, microstructural changes can appear very rapidly, cf. Hallberg et al. (2007). The observations just described can be seen as providing the rationale for the dependency of the strength of the QT material on temperature as shown in Fig. 3.

The temperature-dependence of the specific heat $c$ was chosen as $c=c_{1}+c_{2}\left(\theta-\theta_{0}\right)$ in relation to (12), yielding the two parameters $c_{1}$ and $c_{2}$. In line with Mapelli et al. (2005), these parameters were given the values of $c_{1}=584.13 \mathrm{~J} / \mathrm{kgK}$ and $c_{2}=0.116 \mathrm{~J} / \mathrm{kgK}^{2}$, applying to $100 \mathrm{Cr} 6$ steel.

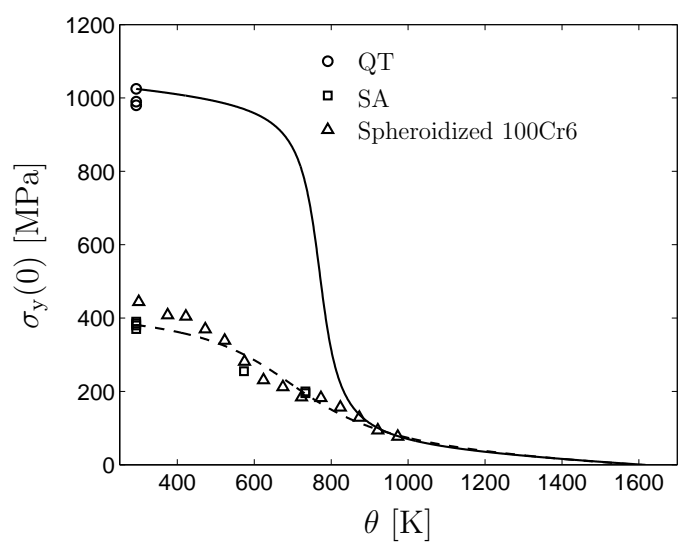

Figure 3: Temperature dependence of the initial yield stress. The experimental results (symbols) are shown together with the fit of the present model for the QT (solid line) and the SA (dashed line) materials, respectively. Results for spheroidized 100Cr6, taken from Löwisch and Dalgic (2006), are shown for comparison. 


\subsection{Hardening behavior}

Note in connection with the mechanical response of the material shown in Fig. 1 that for the SA material there is a very distinct yield stress level at which discontinuous yielding occur, this creating a plateau in the yield curve, a behavior that is largely lacking in the QT material. It can be assumed for both materials that $\hat{\sigma}_{\mathrm{y}}\left(\varepsilon_{\mathrm{eff}}^{v p}\right)=\hat{\sigma}_{\mathrm{y}}(0)+\hat{H}\left(\varepsilon_{\mathrm{eff}}^{v p}\right)$. To capture the plateau effect, the hardening term $\hat{H}$ is constructed with use of both a linear and a nonlinear term. This results in a discontinuous hardening behavior of the form

$$
\begin{aligned}
& \hat{H}=H_{1} \varepsilon_{\text {eff }}^{v p} \quad \text { if } \varepsilon_{\text {eff }}^{v p}<\varepsilon_{\text {shift }}^{v p} \\
& \hat{H}=R_{\infty}\left[1-\exp \left(\frac{H_{2}}{R_{\infty}}\left(\varepsilon_{\text {shift }}^{v p}-\varepsilon_{\text {eff }}^{v p}\right)\right)\right] \quad \text { if } \quad \varepsilon_{\text {eff }}^{v p} \geq \varepsilon_{\text {shift }}^{v p}
\end{aligned}
$$

where $\varepsilon_{\text {shift }}^{v p}$ denotes the viscoplastic strain at which the hardening shifts from being linear to nonlinear. In (31) $R_{\infty}$ represents the saturation hardening, $H_{1}$ and $H_{2}$ being the linear and nonlinear hardening moduli, respectively. Fig. 4 shows the response according to the model, account being taken of heat generation which is discussed later on, calibrated to experimental data obtained at a strain rate of $0.14 \mathrm{~s}^{-1}$. For the SA material, parameter values of $H_{1}=300 \mathrm{MPa}, H_{2}=16000 \mathrm{MPa}$ and $R_{\infty}=400 \mathrm{MPa}$ are selected, the corresponding values for the QT material being taken as $H_{2}=8500 \mathrm{MPa}$ and $R_{\infty}=280 \mathrm{MPa}$, respectively. For the SA material, the plastic strain at which the hardening behavior changes from being linear to nonlinear is $\varepsilon_{\text {shift }}^{v p}=0.0065$, whereas for the QT material $\varepsilon_{\text {shift }}^{v p}=0$ since continuous yielding is assumed, making the parameter $H_{1}$ redundant in this case.

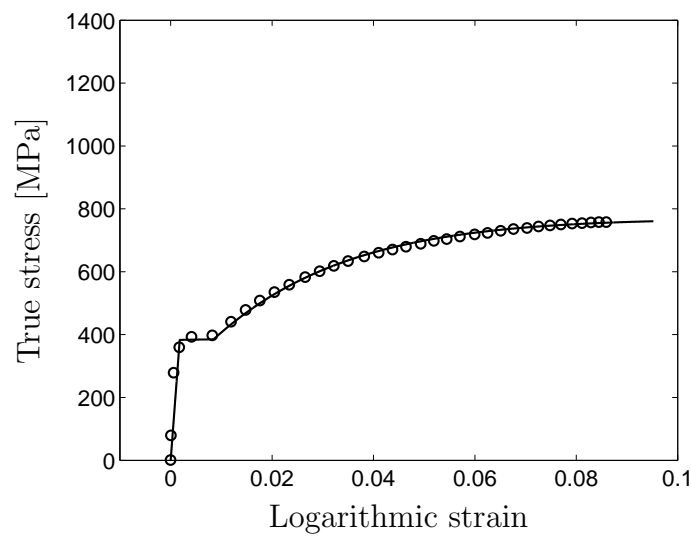

(a) $\mathrm{SA}$

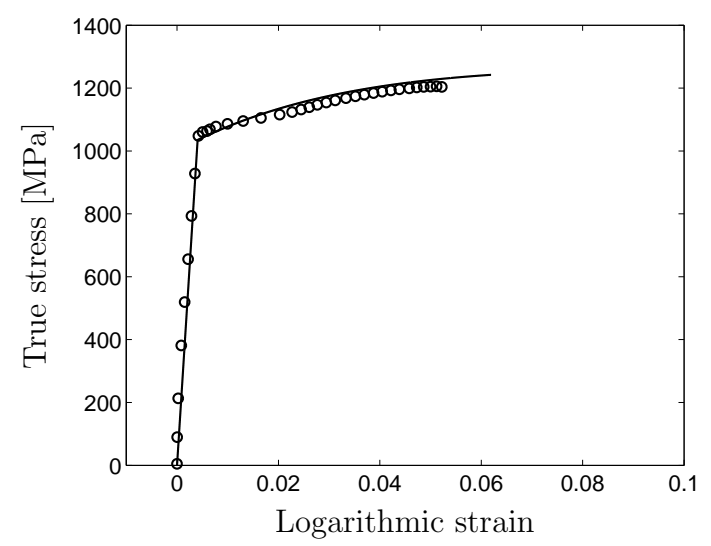

(b) QT

Figure 4: Response according to the model (solid lines) calibrated against experimental results (circles) obtained at a strain rate of $0.14 \mathrm{~s}^{-1}$. Note the discontinuous initial hardening of the SA material. 


\subsection{Stored energy of cold work}

During deformation, the internal energy of a solid changes. In metals and steel, thermoelastic coupling leads to a slight decrease in temperature during the initial elastic deformation, the temperature decrease reaching its lowest value shortly after the point at which inelastic deformation is initiated. During the plastic, i.e. irreversible, deformation mechanical dissipation occurs, this to a large extent being the source of an internal heating that rapidly reverses the thermoelastic trend and produce an increase in temperature. The remainder of the internally expended energy is stored in the material at a microscopic level through micro-cracking and the creation and rearrangement of such material imperfections as point defects, stacking faults, twins and dislocation pile-ups. These microstructural alterations can also include phase transformations, as discussed by Hallberg et al. (2007). The stored energy of cold work is related mainly to changes in dislocation density within the material. It is common for steel to have an initial dislocation density (length of dislocation line per unit volume) of $10^{10}-10^{12} \mathrm{~m}^{-2}$, which after extensive plastic deformation increases to $10^{15}-10^{16} \mathrm{~m}^{-2}$. At sufficient plastic deformation the dislocation density becomes saturated, the material being incapable of further storage of cold-work energy. The massive increase in dislocation density that occurs involves a significant increase in strain energy within the material, which leads to the deformed solid becoming thermodynamically unstable. The return to a more stable state through a lowering of the internal energy level can occur through thermally activated processes such as cross-slip and diffusion. These are dynamic processes, however, that result in a time-dependent stabilization. Reversal of the cold-work effects occurs through a combination of recovery, recrystallization and grain growth.

In the present work, $\rho_{0} \psi^{v p}$ represents the stored energy of cold work. It is a measure of the storage of energy or accumulated energy in the material at the microscopic level. Assuming $\psi^{v p}$ to depend on the temperature would lead to inaccurate predictions, namely that a change in temperature without any accompanying changes in the microstructure of the material would cause the stored energy level to change. Accordingly, it is natural to assume that $\psi^{v p}$, as used previously, is a function of an internal variable $\kappa$ only. A variable that evolve during viscoplastic loading. The evolution of $\kappa$ will, however, obviously depend on the temperature.

It has been shown by Bever et al. (1973) for example, that depending on the temperature, different graphs of stored energy of cold work versus plastic strain are obtained. Thus, identifying $\varepsilon_{\text {eff }}^{v p}$ with $\kappa$ would lead to the contradiction just discussed. This is, in the present model, a consequence of letting the stored energy of cold work be controlled by a single, "macroscopic", scalar internal variable. If additional internal variables were to be introduced - e.g. variables describing the state of the microstructure - further possibilities would be available. As will be shown, however, the results reported by Bever et al. (1973) can be reproduced by the approach taken here without violating the principle of the stored 
energy being a function of one evolving internal variable only.

In the present coupled thermomechanical analysis it is of interest to quantify how much of the dissipated energy is converted into heat and to what extent it is stored in the microstructure of the material. To this end, the rate of viscoplastic work is defined as

$$
\dot{W}^{v p}=\boldsymbol{\tau}: \boldsymbol{d}^{v p}
$$

The mechanical dissipation in (7) can be formulated then as

$$
\mathcal{D}_{\text {mech }}=\dot{W}^{v p}-R \dot{\kappa} \geq 0
$$

Internal heat generation is traditionally calculated as the rate of plastic work multiplied by a constant factor $\beta$, e.g. Simo and Miehe (1992) and Wriggers et al. (1992). Such an approach states that

$$
\mathcal{D}_{\text {mech }}=\beta \dot{W}^{v p}
$$

implying that $\beta$ controls the fraction of plastic work that generates heat. The fraction of plastic work in the material that is stored by cold work is controlled then by the factor $1-\beta$. Rearranging (34) and using (33), it is found that

$$
\beta=1-\frac{R \dot{\kappa}}{\dot{W}^{v p}} \Rightarrow \rho_{0} \dot{\psi}^{v p}=R \dot{\kappa}=(1-\beta) \dot{W}^{v p}
$$

By use of (9) it can thus be determined that $R \dot{\kappa}$ in fact controls the rate of energy storage. From (34) is can also be concluded that the principle $\beta \geq 0$ must hold in order for the dissipation inequality to be fulfilled, $\beta$ being defined in (35a). In order to choose a model and evaluate $\beta, g_{R}$ needs to be identified. In the present case, in order to avoid introducing additional functions, the following simple choice is made of

$$
g_{R}=R T(\theta)
$$

It follows from (18b) then that

$$
\dot{\kappa}=T(\theta) \dot{\varepsilon}_{\mathrm{eff}}^{v p}
$$

Use of expression (35a) and of the principle that $\dot{W}^{v p}=\sigma_{\text {eff }} \dot{\varepsilon}_{\text {eff }}^{v p}$ allows $\beta$ to be identified as

$$
\beta=1-\frac{R}{\hat{\sigma}_{\mathrm{y}}\left(\varepsilon_{\mathrm{eff}}^{v p}\right)+\hat{\sigma}_{\mathrm{y}}(0)\left(\eta \dot{\varepsilon}_{\mathrm{eff}}^{v p}\right)^{1 / n}}
$$

advantage being taken of (25) here. The final task is to identify $R$ on the basis of experimental data. Taking account of experimental evidence and following Håkansson et al. (2005) and Ristinmaa et al. (2007) for example, it can first be noted that with use of (4) and (11) the heat equation (5) can be written as

$$
\rho_{0} c \dot{\theta}=\dot{W}^{v p}-\rho_{0} \frac{\partial \psi^{v p}}{\partial \kappa} \dot{\kappa}-\frac{3 K \alpha}{J^{r}} \dot{J}^{r}
$$


In the following, the last term - that of the thermoelastic effects - is ignored since it is rapidly overshadowed by the heating produced by the plastic dissipation, cf. Bever et al. (1973) for example. The quantity $\psi^{v p}(\kappa)$ in (9), which defines $R$, can then be obtained on the basis of experiments, making use of (39) rewritten as

$$
\rho_{0} \psi^{v p}=\int_{0}^{\kappa} \frac{1}{T}\left(\sigma_{\mathrm{eff}}-\frac{\rho_{0} c \dot{\theta}}{\dot{\varepsilon}_{\mathrm{eff}}^{v p}}\right) d \kappa
$$

Note that the quantities needed can be obtained from a single uniaxial tensile test at constant strain rate, cf. Rosakis et al. (2000). Fig. 5 shows the stored energy of cold work obtained from the tensile tests performed in the present study. The measurement of stored energy involves the rate of temperature change, making the readings very sensitive to thermal disturbances. This causes the scatter in the experimental results as seen in Fig. 5 and no certain conclusion on the temperature dependence of the stored energy - i.e. of $\kappa$ - can be drawn from the available results. The current experimental findings are used only to approximately determine the initial slope of the stored energy curve. Only results regarding the SA material is shown since the appearance of the QT data is similar. As discussed earlier the amount of stored energy of cold work the material contains reaches a saturation value when the plastic deformation of the material has become sufficiently severe. The saturation value here is expected to be independent of the loading rate, even if the time needed for this threshold to be reached can differ. This allows a single function $\hat{\Psi}$ to be fitted against the stored energy results, cf. Fig. 5. An approximation of the amount of stored energy at extensive plastic deformation can be achieved by taking account of the strain energy caused by individual dislocations, cf. Bever et al. (1973), Kapoor and Nemat-Nasser (1998) and Zehnder (1991). Following the work of Cottrell (1963), the dislocation strain energy $E$ due to a dislocation density of $\rho_{\mathrm{d}}$ is assumed to be described by $E=E_{\text {self }}+E_{\text {core }}$, the dislocation self-energy $E_{\text {self }}$ being given by

$$
E_{\text {self }}=\rho_{\mathrm{d}} \frac{G b^{2}}{4 \pi C} \ln \left(\frac{r}{r_{0}}\right)
$$

In (41) $b$ is the length of the Burgers vector and $C=1$ and $C=1-\nu$ holds for screw and edge dislocations, respectively, $\nu$ being introduced here to denote the Poisson ratio, which is taken to be $\nu=0.3$. In (41) $r$ and $r_{0}$ are the outer and inner radii, respectively, of the individual dislocation strain field. The dislocation strain energy $E_{\text {core }}$ within the coreradius $r_{0}$ is calculated as $E_{\text {core }}=\rho_{\mathrm{d}} G b^{2} / 10$, cf. Smallman and Bishop (1999). In the case of large plastic strains the distance between the individual dislocations is approximated as $1 / \sqrt{\rho_{\mathrm{d}}}$. The outer radius of the dislocation strain field is taken, therefore, as $r=\left(2 \sqrt{\rho_{\mathrm{d}}}\right)^{-1}$ and the inner radius as $r_{0}=b$, cf. Kapoor and Nemat-Nasser (1998) and Zehnder (1991). Assuming then that equal amounts of screw and edge dislocations exists, and ignoring the strain energy contributions from such point defects as vacancies and interstitials, allows 


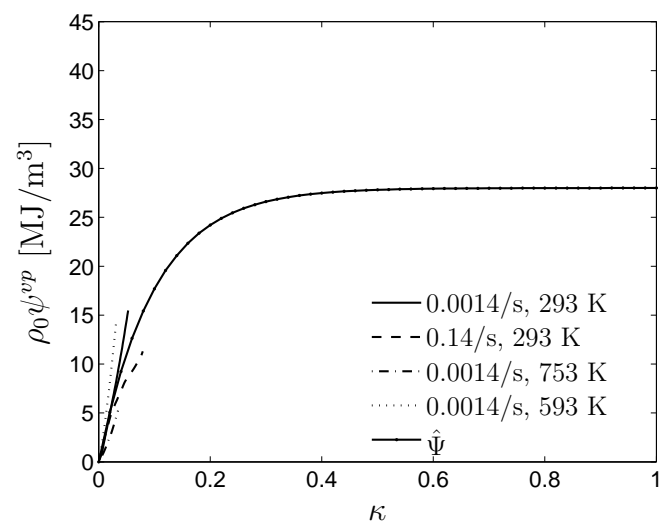

Figure 5: The stored energy of cold work for the SA material obtained in experiments involving different initial temperatures and strain rates. The corresponding results regarding the QT material has the same appearance. $\hat{\Psi}$ denotes the function used in the present model.

an estimate of the stored energy of cold work in the case of large plastic strains to be calculated. The "saturated" dislocation density is taken as $\rho_{\mathrm{d}}=10^{16} \mathrm{~m}^{-2}$ and the length of the Burgers vector as $b=0.3 \mathrm{~nm}$. It was found that small variations in the value of $b$ result only in negligible variations in the energy dissipated as heat. This allows $E \approx 28 \mathrm{MJ} / \mathrm{m}^{3}$ to be calculated. Since only minor differences are seen when comparing the stored energy of the SA and QT materials, respectively, calibration against the SA data is used in both cases, not to clutter the formulation. In line with Ristinmaa et al. (2007), $\hat{\Psi}$ is chosen as

$$
\hat{\Psi}(\kappa)=\frac{r_{1}}{r_{3}\left(r_{2}+r_{3}\right)}\left[r_{2}\left\{\exp \left(r_{3} \kappa\right)-1\right\}+r_{3}\left\{\exp \left(-r_{2} \kappa\right)-1\right\}\right] \exp \left(-r_{3} \kappa\right)
$$

where the values $r_{1}=280.0 \times 10^{6} \mathrm{~J} / \mathrm{m}^{3}, r_{2}=20.5 \times 10^{6}$ and $r_{3}=10.0$ are selected. The $r_{1}$-parameter is based on the rough estimate of the saturation value for the stored energy according to (41). With use of (9) $R$ is then calculated as

$$
R(\kappa)=\frac{d \hat{\Psi}}{d \kappa}=r_{1}\left[1-\exp \left(-r_{2} \kappa\right)\right] \exp \left(-r_{3} \kappa\right)
$$

The present formulation provides a single expression for the stored energy, one which depends on the internal variable $\kappa$ only. It is possible, however, to use the expression (37) to identify, on the basis of $\kappa=\varepsilon_{\mathrm{eff}}^{v p} T(\theta)$, the appearance of the stored energy in arbitrary isothermal cases. The presence of the temperature-dependent function $T$ results in the behavior of the energy stored in the material as the temperature increases being physically sound in the sense of an increase in temperature reducing the amount of energy stored. Fig. 6a shows the amount of energy stored in the SA material at two constant temperatures and Fig. $6 \mathrm{~b}$ shows the corresponding appearance of the heat exchange ratio $\beta$ as given by 
(38). The results for the QT material are again similar and are therefore not shown. Note that the temperature dependence can be adjusted by a different choice of $g_{R}$ in (36).

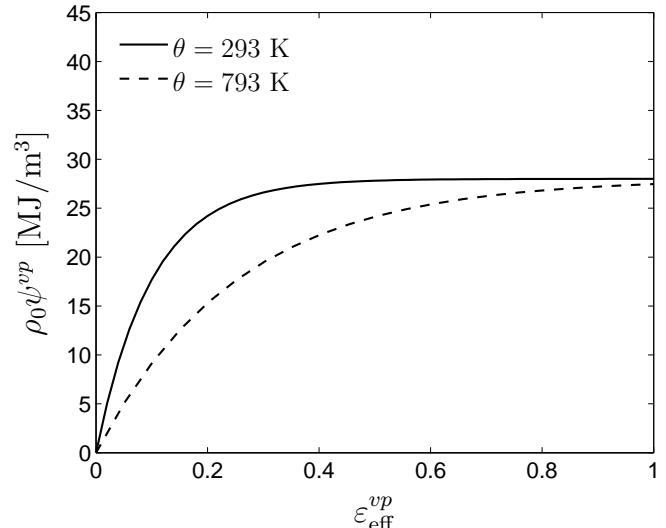

(a)

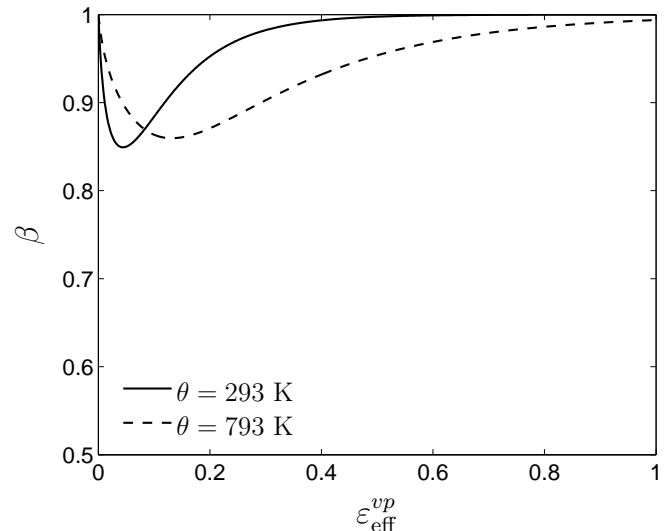

(b)

Figure 6: (a) The stored energy of cold work obtained for the SA material at two different constant temperatures. (b) The heat exchange ratio $\beta$ corresponding to the curves in (a).

\section{Simulation example - Upsetting of an axisymmetric billet}

To indicate the capabilities of the proposed model and illustrate the differences in behavior between the SA and QT materials, simulations of the upsetting of an axisymmetric billet were performed. This is a problem that many authors have considered, e.g. Lin and Brocks (2006), Simo and Miehe (1992) and Tugcu (1996). The geometry of the model is shown in Fig. 7 where the hashed area marks the region which is being simulated, advantage being taken of the symmetry of the problem. In the present simulations, the geometry shown in Fig. 7 is given by $2 h_{0}=11.25 \mathrm{~mm}$ and $2 r_{0}=6 \mathrm{~mm}$. The coefficient of friction between the tool and the workpiece is set to 0.15 . To perform simulations the constitutive model is implemented in the commercial finite element package Abaqus Explicit, in which the geometry is modeled by 6720 axisymmetric and thermomechanically coupled elements of type CAX3T. These three-node triangular elements are employed in a pattern in which four elements that form a quadrilateral share a common node centered in the quadrilateral. Severe deformation of the billet results in adiabatic internal heating of the material as plastic work is dissipated, leading to thermal softening. This thermal softening, however, is coupled with strain hardening of the material as the plastic deformation progresses, both through a rapid increase in the dislocation density and through the dislocations becoming increasingly glissile. A process involving competition between softening and hardening 
thus characterize the material behavior during deformation. In addition, both shear band formation and the localization of deformation are prone to be initiated at the occurrence of any geometric or material irregularities if the thermal softening of the material is sufficient to reverse the stabilizing effects of strain hardening. Additional plastic straining also develops rapidly in the shear band, further promoting heating and localization of the deformation. In Fig. 3 one can note that the initial yield stress of the QT material, as given by the calibration procedure discussed later in more detail, drops markedly as the temperature increases, an effect that is much less pronounced in the SA material. This will lead to shear band formation being much more likely to occur in the hardened material, a tendency that is further supported by the hardening behavior of the materials shown in Fig. 4. The SA material has a substantially lower initial yield stress than the QT material does, yet the additional hardening that occurs as the plastic strain increases is much more marked in the SA material which stabilizes any localization tendencies.
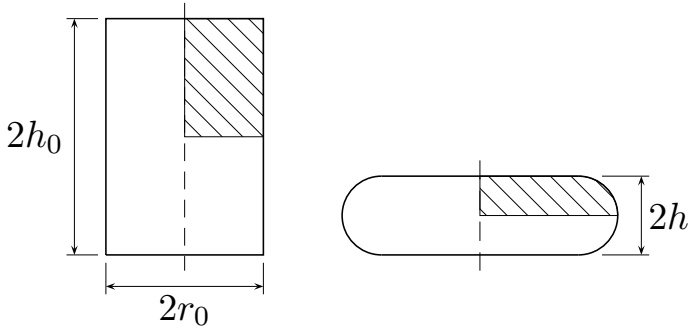

Figure 7: Geometry used in the simulation of the axisymmetric upsetting of a billet. The undeformed geometry is shown at the left and the deformed geometry - with barreling due to friction against the tool - is shown schematically at the right.

\subsection{Comparison with experimental results}

Initial forming experiments were conducted for calibration of the model and verification of the simulation results. In the tests carried out, cylindrical specimens (11.25 $\mathrm{mm}$ in height and $6 \mathrm{~mm}$ in diameter) were deformed using a hydraulic high velocity pressing machine capable of achieving impact velocities of up to $8 \mathrm{~m} / \mathrm{s}$. Force measurements were conducted using a Kistler load ring located below the sample, whereas measurements of the impact velocities, observations of the continuous changes in shape that occurred and of the details of the microstructure were not performed. A damping ring (spheroidize-annealed 100Cr6) was placed around the sample so as to stop the deformation of the sample at a specific point in time. A plastic film was placed both under the sample and on top of it so as to reduce friction. The results of the filtered measurements of force are shown in Fig. 8 together with the simulated material response obtained by use of the present model. The calibrated simulations were found to agree closely with the experimental results. The tendency for 


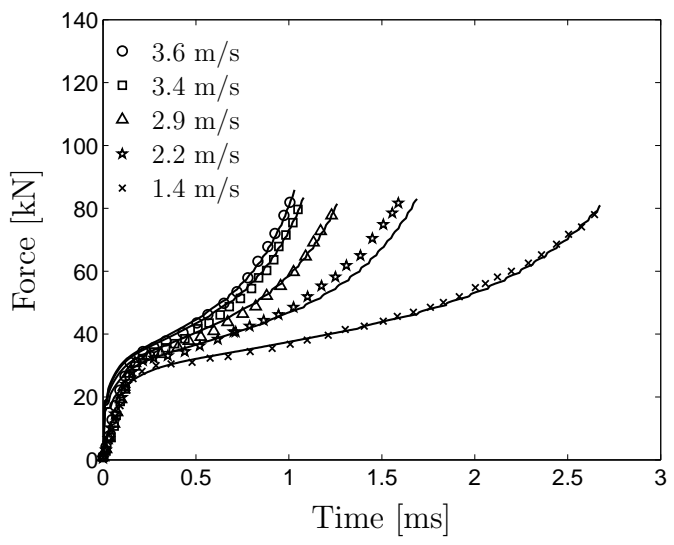

(a) SA

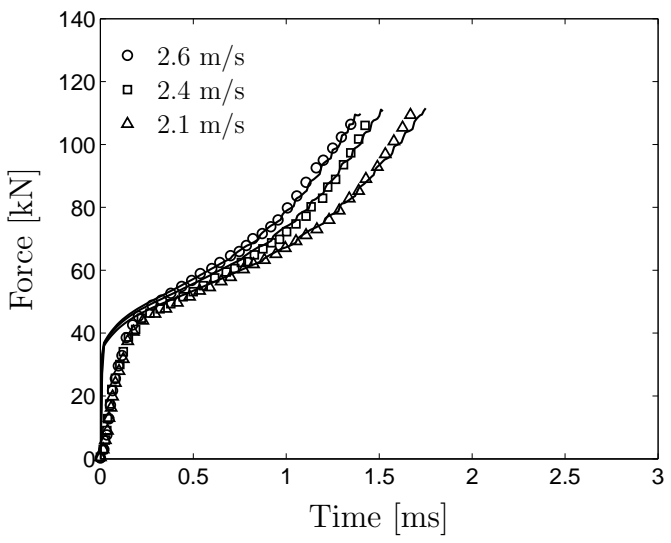

(b) QT

Figure 8: Forming experiments. Simulated response (solid lines) compared with the experimental results (symbols) for different deformation velocities. Note that the velocities of deformation stated here are those used in the simulations as approximations of the mean velocities found in the experiments.

sharp shear bands to be formed in the QT but not in the SA material was evident, as can be seen in Fig. 9. The presence of shear bands was in fact used to calibrate the simulation

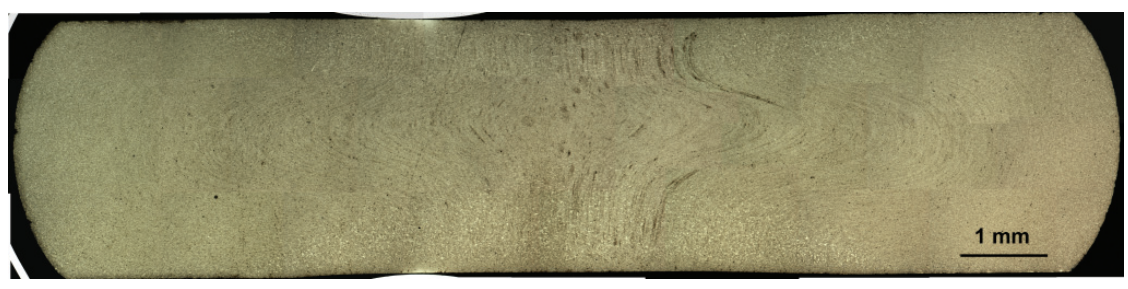

(a) $\mathrm{SA}$

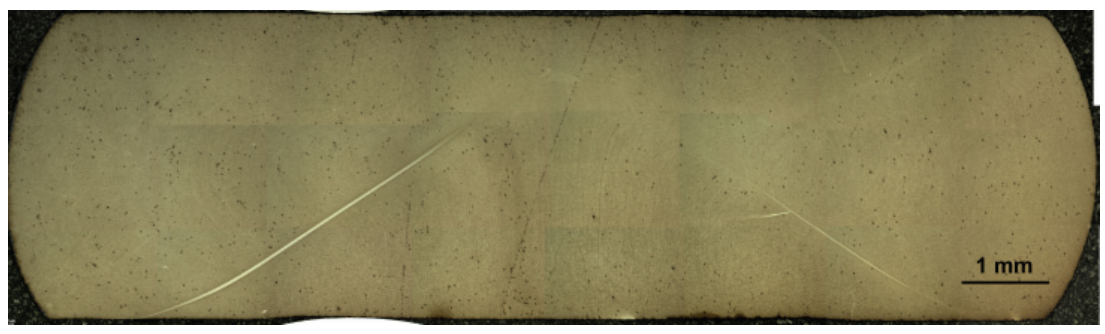

(b) QT

Figure 9: Microscope images of the cross-section of the deformed specimen. (a) The SA material deformed at a rate of approximately $3.5 \mathrm{~m} / \mathrm{s}$. (b) The QT material deformed at a rate of approximately $2.5 \mathrm{~m} / \mathrm{s}$.

model since localization was found to define, rather distinctly, the appearance of the drop 
in initial yield stress as seen in Fig. 3. If the drop is shifted towards higher temperatures, the localization of the deformation in the material is lost. Correspondingly, a shift towards lower temperatures results in too much thermal softening, producing an unsatisfactory fit with the experimental results shown in Fig. 8. Note, however, that the microstructural mechanisms underlying this behavior are somewhat unclear.

The particular simulations shown in Figs. 10 and 11 were conducted by prescribing a constant tool velocity of $3.5 \mathrm{~m} / \mathrm{s}$. This creates a pronounced impact deformation in relation to the dimensions of the blank, comparable to what is found in industrial high velocity metal forming processes. The final height of the deformed workpiece is $2 h=3.5 \mathrm{~mm}$. Fig. 10a shows a simulation of a billet made of SA $100 \mathrm{Cr} 6$ at three stages of deformation. The contours indicate the distribution of effective viscoplastic strain $\varepsilon_{\text {eff }}^{v p}$ in the material. Fig. 10b shows a corresponding simulation for the QT material. In comparing these pictures, clear differences in localization behavior can be noted. The localization of the deformation and the subsequent shear band formation are evident in the case of the QT material, a distinct localization line being visible in the bottom picture as a result of the calibrated QT-curve in Fig. 3. Sharp localization of this sort is lacking in the SA material, in which the thermal softening is much less pronounced, cf. Fig. 3, the strain hardening stabilizing the softening tendency and diffusing the shear band formation. Fig. 11 shows the temperature distribution in the billet for the same states of deformation as in Fig. 10. Since the adiabatic heating is due to the dissipation of internal viscoplastic work, the temperature contours closely resemble those of the distribution of effective viscoplastic strain. Note that in the QT material there is an appreciable temperature increase, one which is responsible for the thermal softening and is largely concentrated within the region of localization. These differing material-dependent deformation modes can be clearly seen in Ryttberg (2007), describing the distinct localization together with subsequent internal fracture found in the QT material.

Fig. 9 shows the deformed specimens to have a characteristic barrel shape which is found in the simulations as well, cf. Figs. 10 and 11. An asymmetry in the localization can be seen in Fig. 9, probably due to varying conditions of friction at the top and bottom surfaces of the specimen. During deformation, the internal temperature of the QT-material can rise to well above the austenitization temperature of approximately $750^{\circ} \mathrm{C}$. This heating, in combination with subsequent rapid and localized cooling, allows reaustenitization and formation of new martensite in the material to take place, cf. Shi and Liu (2006). These microstructural changes commonly appear in connection with localization of the deformation and the formation of so-called white layers or etching bands. The morphology of these white layers is characterized by grains of much smaller size than the in bulk of the material and a corresponding increase in hardness, cf. Ramesh et al. (2005). These microstructural changes, together with white layer formation, have been observed under widely differing strain and temperature conditions, cf. Ramesh et al. (2005). 

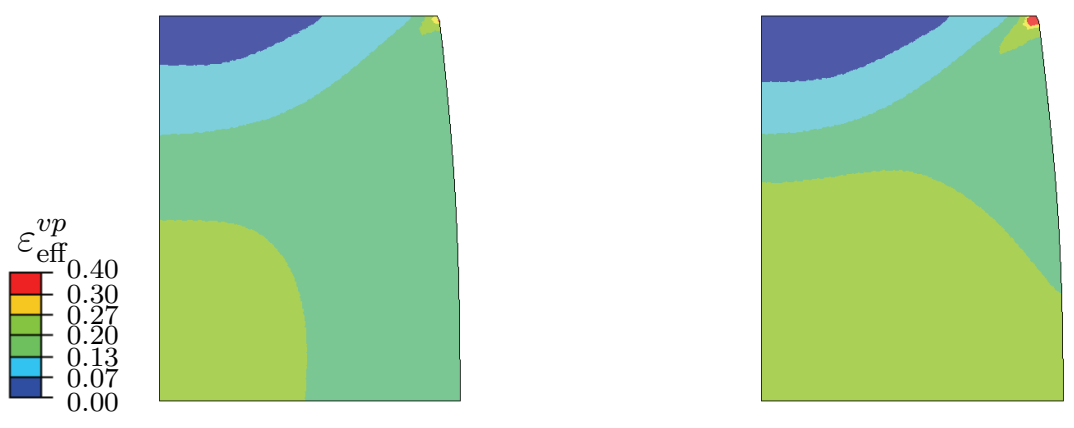

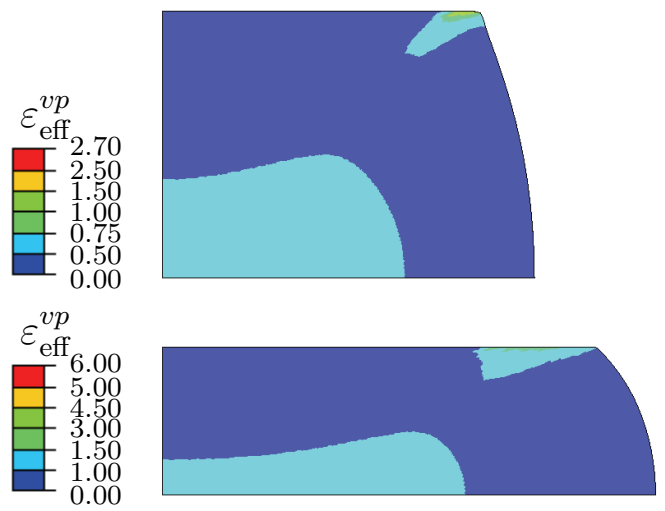

(a) $\mathrm{SA}$
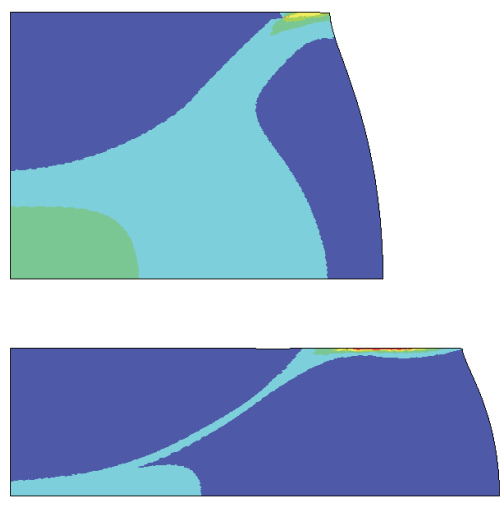

(b) QT

Figure 10: Distribution of effective viscoplastic strain $\varepsilon_{\text {eff }}^{v p}$ at 30,65 and $100 \%$ deformation as obtained in simulations using the present model. 

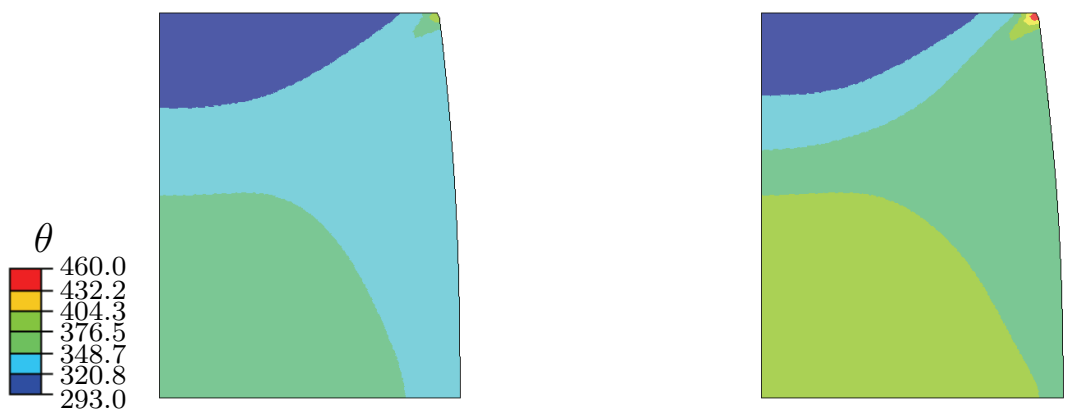

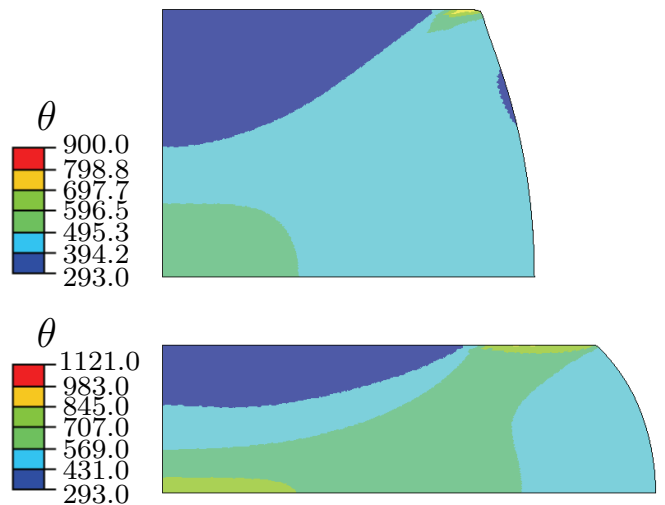

(a) $\mathrm{SA}$
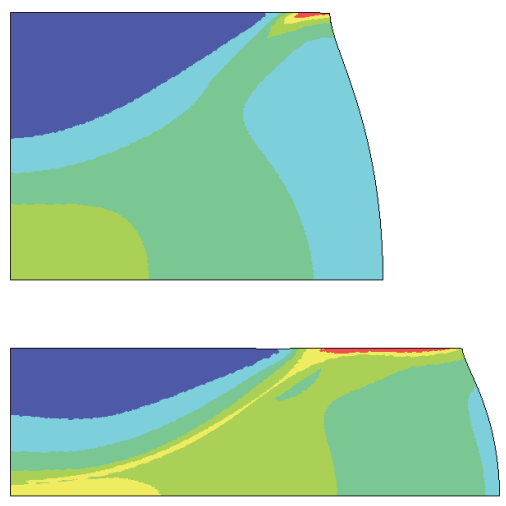

(b) QT

Figure 11: Distribution of absolute temperature $\theta$ at 30, 65 and $100 \%$ deformation as obtained in simulations using the present model. 
Since neither temperature measurements nor close microstructural investigations of the shear bands were performed in the tests conducted here, the presumed rise in temperature within the shear bands could not be verified. However, shear band formation and corresponding microstructure and temperature effects resulting from high velocity parting-off of 100Cr6 have been discussed previously by Ryttberg (2007). Regarding parting-off, extreme shear localization accompanied by grain refinement down to the $\mathrm{nm}$ scale was observed.

In performing simulations involving localization of deformation, as can be seen in Figs. 10 and 11, the severity of the localization is to some extent artificially controlled by the element discretization that was selected. A higher mesh density is generally expected to produce a more sharply defined region of localization, whereas a coarser mesh discretization is expected to make the shear band formation more diffuse or not to occur at all. When compared with the results obtained experimentally, cf. Fig. 9, the element density used in the present simulations was found to provide reasonable results.

\section{Concluding remarks}

A constitutive model, taking account of both rate dependent plasticity and of finite-strain kinematics was formulated within a thermodynamically consistent setting. Thermomechanical coupling was also included in the model by taking account of the adiabatic heating produced by dissipation of internal viscoplastic work. This was achieved by formulating the heat generation and hardening behavior of the material in a consistent fashion based on experimental evidence rather than, as is usually done, postulating the relationships involved a priori. Conversion of the dissipation of internal viscoplastic work into heat is allowed in this way to depend both on the viscoplastic strain that occurs and on the viscoplastic strain rate, instead of disregarding the true material behavior through a constant conversion factor the value of which has been simply assumed. The stored energy of cold work that is imposed in the material due to inelastic deformation is discussed and it is concluded that this stored energy cannot be a function of temperature which would yield unphysical material behavior. The stored energy is instead allowed to vary with an internal variable which, however, can be taken as temperature dependent. In absence of reliable experimental data, the temperature dependence of this internal variable has not been pursued further here although this dependence is possible within the current formulation. Calibration of the proposed model against experimental data regarding 100Cr6 steel in two heat treated conditions allowed simulations of a high velocity forming process to be performed. Significant differences in deformation behavior between samples that had been heat treated differently were observed. Quenched and tempered 100Cr6 underwent a marked softening as the temperature increased, promoting very rapid formation of a distinct adiabatic shear band. Behavior of this type contrasted with that of the spheroidize-annealed material, in which the deformation hardening stabilized the thermal softening and prevented any sharp 
localization of the deformation that occurred.

\section{Acknowledgment}

Håkan Hallberg and Kristina Ryttberg gratefully acknowledge the funding provided by the ProViking program Simu-Net-Shape under grant SFF V03.22.

\section{A Integration of the constitutive relations}

It is possible to handle the integration of the constitutive relations here by defining a system of equations in terms of e.g. the components of $\boldsymbol{F}^{v p}$ and the set of internal variables, obtaining a system of equations to be solved iteratively. An alternative approach was chosen here, seeking greater numerical efficiency. Following Simo (1998), and letting $n$ and $n+1$ denote two successive load steps, an exponential update format was defined as

$$
\boldsymbol{F}_{n+1}^{r}=\boldsymbol{A}^{-1} \boldsymbol{F}^{r, t r i a l} \quad \text { where } \quad \boldsymbol{A}=\exp \left[\Delta \lambda\left(\frac{\partial g}{\partial \boldsymbol{\tau}}\right)_{n+1}\right]
$$

this being used to update the reversible part of the deformation gradient from a trial state - denoted by a superscript trial - to the current state. The elastic trial state is obtained by freezing the plastic flow such that $\boldsymbol{F}_{n+1}^{r, t r i a l}=\boldsymbol{F}_{n+1} \boldsymbol{F}_{n}^{v p-1}$. Taking advantage of (44a) allows the Finger tensor to be written as

$$
\boldsymbol{b}_{n+1}^{r}=\boldsymbol{F}_{n+1}^{r}\left(\boldsymbol{F}_{n+1}^{r}\right)^{T}=\boldsymbol{A}^{-1} \boldsymbol{b}^{r, \text { trial }} \boldsymbol{A}^{-T}
$$

In the case of an elastic material response, i.e. when $f^{\text {trial }}<0$, updating can be performed simply by setting $(\cdot)_{n+1}=(\cdot)^{\text {trial }}$. Viscoplastic loading occurs if $f^{\text {trial }}>0$ and in this case an exponential update of $\boldsymbol{F}^{v p}$ can be performed together with a backward Euler update of $\dot{\varepsilon}_{\text {eff }}^{v p}=\Delta \lambda$. I.e. with (22) and (45) we have the following system of nonlinear equations:

$$
\begin{aligned}
& \mathcal{R}_{\boldsymbol{b}^{r}}=\boldsymbol{b}_{n+1}^{r}-\boldsymbol{A}^{-1} \boldsymbol{b}^{r \text { trial }} \boldsymbol{A}^{-T}=\mathbf{0} \\
& \mathcal{R}_{\Delta \lambda}=\eta \frac{\Delta \lambda}{\Delta t}-h=0
\end{aligned}
$$

The eigenvalue decomposition theorem provides a numerically efficient way of dealing with these equations. Since the eigenvectors of the quantities involved coincide, taking the logarithm of (46a) results in

$$
\ln \left(\boldsymbol{V}^{r}\right)^{d e v}=\ln \left(\boldsymbol{V}^{r, t r i a l}\right)^{d e v}-\Delta \lambda \frac{\partial g}{\partial \boldsymbol{\tau}}
$$

advantage being taken here of the fact that $\frac{\partial g}{\partial \tau}$ is purely deviatoric, yielding $J^{r}=J^{r \text { trial }}=$ $\operatorname{det}\left(\boldsymbol{V}^{r}\right)$. On the basis of (14), and taking advantage of (20b), it is now found that

$$
\boldsymbol{\tau}^{\text {dev }}=\boldsymbol{\tau}^{\text {dev,trial }}-3 G \Delta \lambda \frac{\boldsymbol{\tau}^{\text {dev }}}{\sigma_{\text {eff }}} \quad \text { where } \quad \boldsymbol{\tau}^{\text {dev,trial }}=2 G \ln \left(\boldsymbol{V}^{r, \text { trial }}\right)^{\text {dev }}
$$


This expression can be further reduced to the format

$$
\sigma_{\text {eff }}=\sigma_{\text {eff }}^{\text {trial }}-3 G \Delta \lambda \quad \text { where } \quad \sigma_{\text {eff }}^{\text {trial }}=\left(\frac{3}{2} \tau^{\text {dev,trial }}: \boldsymbol{\tau}^{\text {dev,trial }}\right)^{1 / 2}
$$

which enables the yield function $f$ to be handled conveniently in the updating scheme and thus the overstress function $h$ as well. This allows the equations in (46) to be reduced to the single scalar equation

$$
\mathcal{R}(\Delta \lambda)=\eta \frac{\Delta \lambda}{\Delta t}-h=0 \quad \text { where } \quad h=\left\langle\frac{\sigma_{\mathrm{eff}}^{\text {trial }}-3 G \Delta \lambda-\sigma_{\mathrm{y}}\left(\varepsilon_{\mathrm{eff}, n}^{v p}+\Delta \lambda, \theta\right)}{\sigma_{\mathrm{y}}(0, \theta)}\right\rangle^{n}
$$

The only unknown then is $\Delta \lambda$, which permits a Newton-Raphson scheme to be employed in obtaining a solution. Regarding the thermal field, it is indicated in (5) and (7) that the rate of temperature change can be written as

$$
\dot{\theta}=\frac{\mathcal{D}_{\text {mech }}}{\rho_{0} c}
$$

Once the stored energy term $\psi^{v p}$ that appears in $\mathcal{D}_{\text {mech }}$ has been calculated on the basis of experimental results it is possible - as has already been shown - to perform a simple backward Euler update of the temperature in accordance with $\theta_{n+1}=\theta_{n}+\Delta \theta$. Note that the temperature updating procedure involves direct use of the increment of the viscoplastic multiplier $\Delta \lambda$.

\section{References}

Batra, R. C. and Liu, D.-S. (1990). Adiabatic shear banding in dynamic plane strain compression of a viscoplastic material. International Journal of Plasticity, 6, 231-246.

Batra, R. C. and Stevens, J. B. (1998). Adiabatic shear bands in axisymmetric impact and penetration problems. Computer Methods in Applied Mechanics and Engineering, 151, $325-342$.

Bever, M. B., Holt, D. L., and Titchener, A. L. (1973). The stored energy of cold work. Pergamon Press, first edition.

Chrysochoos, A., Maisonneuve, O., Martin, G., Caumon, H., and Chezeaux, J. C. (1989). Plastic and dissipated work and stored energy. Nuclear Engineering and Design, 114, 323-333.

Clifton, R. J., Duffy, J., Hartley, K. A., and Shawki, T. G. (1984). On critical conditions for shear band formation at high strain rates. Scripta Metallurgica, 18, 443-448. 
Cottrell, A. H. (1963). Dislocations and plastic flow in crystals. Oxford University Press, fifth edition.

Håkansson, P., Wallin, M., and Ristinmaa, M. (2005). Comparison of isotropic hardening and kinematic hardening in thermoplasticity. International Journal of Plasticity, 7, $1435-1460$.

Hallberg, H., Håkansson, P., and Ristinmaa, M. (2007). A constitutive model for the formation of martensite in austenitic steels under large strain plasticity. International Journal of Plasticity, 23, 1213-1239.

Hartley, K. A., Duffy, J., and Hawley, R. H. (1987). Measurement of the temperature profile during shear band formation in steels deforming at high strain rates. Journal of the Mechanics and Physics of Solids, 35(3), 283-301.

Heeres, O. M., Suiker, A. S. J., and de Borst, R. (2002). A comparison between the Perzyna viscoplastic model and the Consistency viscoplastic model. European Journal of Mechanics A/Solids, 21, 1-12.

Hodowany, J., Ravichandran, G., Rosakis, A. J., and Rosakis, P. (2000). Partition of plastic work into heat and stored energy in metals. Experimental Mechanics, 40(2), 113-123.

Kapoor, R. and Nemat-Nasser, S. (1998). Determination of temperature rise during high strain rate deformation. Mechanics of Materials, 27, 1-12.

Kröner, E. (1960). Allgemeine Kontinuumstheorie der Versetzungen und Eigenspannungen. Arch. Rational Mech. Anal., pages 273-334.

Lee, E. H. and Liu, D. T. (1967). Finite strain plasticity particularly for plane waves. Journal of Applied Physics, 38, 19-27.

Lin, R. C. and Brocks, W. (2006). On a finite-strain viscoplastic law coupled with anisotropic damage: theoretical formulations and numerical applications. Archive of Applied Mechanics, 75, 315-325.

Löwisch, G. and Dalgic, M. (2006). The influence of the temperature on the stress-strain hardening curves of a bearing steel and a case hardening steel. Materialwissenschaft und Werkstofftechnik, 37(1), 134-141.

Lubliner, J. (1972). On the thermodynamic foundation of non-linear solid mechanics. International Journal of Non-Linear Mechanics, 7, 237-254.

Manjoine, M. J. (1944). Influence of rate of strain and temperature on yield stresses of mild steel. Journal of Applied Mechanics - Transactions of the ASME, 66, A-211-A-218.

DOI: 10.1061/ASCE0733-93992009135:4345 
Mapelli, C., Venturini, R., and Boniardi, M. (2005). Simulation and optimisation of an annealing process of sub-critical spheroidization annealing of a $100 \mathrm{Cr} 6$ steel. Scandinavian Journal of Metallurgy, 34, 192-204.

Marchand, A. and Duffy, J. (1988). An experimental study of the formation process of adiabatic shear bands in a structural steel. Journal of the Mechanics and Physics of Solids, 36(3), 251-283.

Mason, J. J., Rosakis, A. J., and Ravichandran, G. (1994). On the strain and strain rate dependence of the fraction of plastic work converted to heat: an experimental study using high speed infrared detectors and the Kolsky bar. Mechanics of Materials, 17, $135-145$.

Miller, A. (1987). Unified Constitutive Equations for Creep and Plasticity, pages 139-269. Elsevier Applied Science.

Oliferuk, W., Gadaj, S. P., and Grabski, M. W. (1985). Energy storage during the Tensile Deformation of Armco Iron and Austenitic Steel. Materials Science and Engineering, 70, 131-141.

Olszak, W. and Perzyna, P. (1969). On thermal effects in viscoplasticity. Zeitschrift für angewandte Mathematik und Physik, 20(5), 676-680.

Ottosen, N. S. and Ristinmaa, M. (2005). The Mechanics of Constitutive Modeling. Elsevier, first edition.

Perzyna, P. (1966). Fundamental problems in viscoplasticity. Advances in Applied Mechanics, 9, 243-377.

Phillips, A. and Wu, H.-C. (1973). A theory of viscoplasticity. International Journal of Solids and Structures, 9, 15-30.

Ramesh, A., Melkote, S. N., Allard, L. F., Riester, L., and Watkins, T. R. (2005). Analysis of white layers formed in hard turning of AISI 52100 steel. Materials Science and Engineering A, 390, 88-97.

Ristinmaa, M. and Ottosen, N. S. (2000). Consequences of dynamic yield surface in viscoplasticity. International Journal of Solids and Structures, 37, 4601-4622.

Ristinmaa, M., Wallin, M., and Ottosen, N. S. (2007). Thermodynamic format and heat generation of isotropic hardening plasticity. Acta Mechanica. 
Rosakis, P., Rosakis, A. J., Ravichandran, G., and Hodowany, J. (2000). A thermodynamic internal variable model for the partition of plastic work into heat and stored energy in metals. Journal of the Mechanics and Physics of Solids, 48, 581-607.

Runesson, K., Ristinmaa, M., and Mähler, L. (1999). A comparison of viscoplasticity formats and algorithms. Mechanics of Cohesive-Frictional Materials, 4, 75-98.

Ryttberg, K. (2007). Fracture behaviour and related microstructures of tool steels partedoff at high rates of strain. Licentiate Dissertation, Chalmers University of Technology, Department of Materials and Manufacturing Technology, Sweden.

Sherby, O. D., Lytton, J. L., and Dorn, J. E. (1957). Activation energies for creep of high-purity aluminum. Acta Metallurgica, 5, 219-227.

Shi, J. and Liu, C. R. (2006). On predicting chip morphology and phase transformation in hard machining. International Journal of Advances in Manufactering Technology, 27, 645-654.

Simo, J. C. (1998). Handbook of Numerical Analysis, volume IV, pages 183-499. Elsevier.

Simo, J. C. and Hughes, T. J. R. (2000). Computational Inelasticity. Springer-Verlag, second edition.

Simo, J. C. and Miehe, C. (1992). Associative coupled thermoplasticity at finite strains: Formulation, numerical analysis and implementation. Computer Methods in Applied Mechanics and Engineering, 98, 41-104.

Smallman, R. E. and Bishop, R. J. (1999). Modern Physical Metallurgy and Materials Engineering. Butterworth-Heinemann, sixth edition.

Taylor, G. I. and Quinney, H. (1934). The latent energy remaining in a metal after cold working. Physica Status Solidi, A143, 307-326.

Trötzschel, J., Müller, S., Pantleon, W., and Klimanek, P. (1997). Investigation of local strain fields during high-speed deformation. Computational Materials Science, 9, 207217.

Tugcu, P. (1995). Heat conduction effects on strain localization in plane-strain tension. International Journal for Numerical Methods in Engineering, 38, 2083-2099.

Tugcu, P. (1996). Thermomechanical analysis of upsetting of a cylindrical billet. Computers \& Structures, 58(1), 1-12. 
Wriggers, P., Miehe, C., Kleiber, M., and Simo, J. C. (1992). On the coupled thermomechanical treatment of necking problems via finite element methods. International Journal for Numerical Methods in Engineering, 33, 869-883.

Zehnder, A. T. (1991). A model for the heating due to plastic work. Mechanics Research Communications, 18(1), 23-28.

Zhu, Z. G. and Batra, R. C. (1992). Consideration of phase transformations in the study of shear bands in a dynamically loaded steel block. Transactions of the ASME, 114, $368-377$. 